ing incidence of adult TB cases in 2012, and was related to the economic crisis in Europe. The best strategy for TB case detection in children is contact investigation allowing early diagnosis, which, in turn, allows the implementation of the prophylactic treatment of TB infection, provides successful treatment outcomes, and prevents death. Current molecular diagnostic methods of Mycobacterium tuberculosis (Mbt) usually provide limited information that is often not sufficient for the local outbreak and transmission investigations. Implementation of the modern approaches such as Next generation sequencing technologies in the epidemiological studies of childhood TB has a potential to combine TB diagnosis, drug resistance profiling and epidemiological analysis into one test helping to initiate personalized treatment for every patient timely and correctly.

Case report. A patient, 29 years old, was diagnosed with the $3^{\text {rd }}$ TB episode in her life in 2017. Mbt cultures were obtained and genotyped. Molecular genotyping results showed different spoligo and IS6110 RFLP patterns for all three TB episodes in years 2001, 2011, and 2017.

Epidemiological anamnesis revealed that the first TB episode at the 14 years of age in patient was identified in 2001 during household contact investigation - patient's uncle was diagnosed with TB in 2001. Uncle had TB relapse in 2006. Genotyping results of the uncle's both Mbt cultures obtained in 2001 and 2006, and patient's Mbt culture obtained in 2011 revealed the identical spoligotype (SIT1) and IS6110 pattern with 17 bands for both patients. These results indicated the high possibility of the transmission in the household contact. However, genotyping results from patient's Mbt culture obtained in 2017 showed different genotype.

Whole genome sequencing (WGS) was used for indepth characterisation of $M$. tuberculosis isolates associated with matched pairs of TB cases. The obtained results were in accordance to the genotyping and drug resistance. In addition, the obtained data provided additional resolution of the microevolution of Mbt subpopulations.

The addition of WGS to the epidemiological data and social network analysis could improve the confirmation of the epidemiological links and evaluation of the transmission dynamics of TB. Additionally, rapid WGS data can be used to identify molecular evidence for strain-specific phenotypic variability including anti-mycobacterial drug resistance, further providing rapid onset of appropriate treatment.

This study was supported by Latvian National Research program VPP "BIOMEDICINE".

\subsection{2 doi: 10.15789/2220-7619-2018-4-6.42 MOLECULAR EPIDEMIOLOGY OF TUBERCULOSIS IN LATVIA}

R. Ranka ${ }^{1,2}$, I. Pole ${ }^{1,3}$, S. Markovska ${ }^{1}$, I. Ozere Oz, $^{, 3}$,

V. Riekstina ${ }^{3}$, I. Norvaisa ${ }^{3}$

${ }^{1}$ Latvian Biomedical Research and Study Centre, Riga, Latvia; ${ }^{2}$ Riga Stradinš University, Riga, Latvia; ${ }^{3}$ Riga East University Hospital, Centre of Tuberculosis and Lung Diseases, Riga, Latvia

Tuberculosis is still one of the major infectious diseases in Latvia, causing serious health problems. While the incidence of the disease has steadily declined in the country since year 2001, the rates of drug-resistant tuberculosis are among the highest within the European Union. Molecular genotyping of M. tuberculosis plays an important role both in clinical studies and in the epidemiological investigations, allowing to describe and char- acterize pathogen's population structure. Our previous studies have shown that in Riga and Riga region the majority of $M$. tuberculosis isolates belonged to lineage 4 (Euro-American) and lineage 2 (East-Asia). The family distribution of the isolates comprised 25\% Beijing, 27\% $\mathrm{T}$, and 25\% LAM (Latin-American Mediterranean) isolates, while Haarlem, Ural, and $\mathrm{X}$ families were detected in 11,6 , and $3 \%$, respectively. A high proportion of Beijing and LAM isolates is alarming, as these M. tuberculosis genotypes have been often associated with remarkable pathogenic features such as drug resistance and increased transmissibility. TB incidence in the Latvian region Latgale seems to be higher than the average, and in-depth studies of $M$. tuberculosis isolates in this region could provide additional resolution for the characterization of the lineages circulating in the country. The Latgale region borders with Lithuania in the South, Belarus in Southeast, and Russian Federation in the East. M. tuberculosis isolates in this region were studied by the Spoligotyping and IS6110 RFLP genotyping methods. In total, $56(73.7 \%)$ samples of 76 bacteriologically confirmed TB cases in the year 2017 were available for molecular analysis. The results showed that $52 \%$ of isolates could be classified as common genotypes in Latvia (SIT1, SIT42, SIT50, SIT53, SIT254, SIT262, SIT283, SIT1292), while $48 \%$ of isolates belonged to SITs which are rarely found in the country or were unique (SIT45, SIT47, SIT52, SIT65, SIT118, SIT150, SIT278, SIT1175, SIT1451). The most common spoligotype belonged to the T1 lineage (SIT53, 16\%) followed by SIT1, SIT47 and SIT254 (9\% each). Within all samples studied, 14 isolates (25\%) formed 4 different clusters with 3-5 members in each. The epidemiological links were confirmed for nine patients in 3 clusters (SIT47, SIT65, and SIT1292). When the prevalence of different spoligotypes was analysed between different countries, a similarity between particular genotypes in Latvia and neighbouring countries was observed. In-depth analysis of these isolates on the international scale could be very useful in order to investigate the possible transmission dynamics of $M$. tuberculosis strains.

This study was supported by the Latvian National Research program VPP “BIOMEDICINE”.

\subsection{3} doi: 10.15789/2220-7619-2018-4-6.43

\section{FUNCTIONAL RELEVANCE OF MYCOBACTERIUM TUBERCULOSIS DIVERSITY: FROM GENOTYPES TO IMMUNE RESPONSES AND DISEASE SEVERITY}

\section{Saraiva}

i3S - Instituto de Investigação e Inovação em Saúde, Porto, Portugal; IBMC - Instituto de Biologia Molecular e Celular, Universidade do Porto, Porto, Portugal

The genetic diversity of tuberculosis (TB)-causing bacteria has surprised us over recent years. A growing body of evidence attributes a functional relevance to this diversity, both at the clinical and immune response levels. Investigating the full diversity of Mycobacterium tuberculosis in nature is however impossible. We recently moved from the study of limited collections of M. tuberculosis to an oriented approach, aimed at covering a representation of M. tuberculosis heterogeneity. For this, we studied over 600 TB patients in Porto and over 300 matching M. tuberculosis isolates. We show a highly homogeneous phylogenetic structure of $M$. tuberculosis, with nearly all cases belonging to Lineage 4 (L4). Within the L4 clade, the most represented sublineage was LAM. This host-pathogen sympatric distribution was however shak- 